\title{
Evaluación de consorcios fúngicos nativos para biolixiviar los metales pesados bario, vanadio y cobre presentes en sedimentos de la laguna de Colta del Cantón Colta
}

\section{Evaluation of native fungal consortia to biolixiviar the heavy metals barium, vanadium and copper present in sediments of the Colta's lagoon}

\author{
Anabel Cristina Cáceres del Salto. ${ }^{1}$, Edwin Fernando Basantes Basantes. ${ }^{2}$, Laura Susana \\ Cocha Telenchana. ${ }^{3} \&$ Manuel Patricio Clavijo Cevallos. ${ }^{4}$
}

Recibido: 14-07-2019 / Revisado: 23-07-2019 /Aceptado: 15-08-2019/ Publicado: 10-09-2019

\begin{abstract}
DOI: https://doi.org/10.33262/cienciadigital.v3i3.4.871

The physical-chemical and microbiological characterization of the sediment, isolation and purification of fungal strains is performed by means of successive planting techniques.

The macroscopic and microscopic characterization of the strains was carried out, different selection tests were carried out: antagonism and identification for subsequent massification and inoculation in the sediment, the process was executed to obtain the native fungal consortia of the sediments from the Colta`s lagoon. Using equipment such as: Horizontal laminar flow chamber, autoclave, incubator, inductively coupled plasma (ICP), stove, among the main ones.

At the end of the selection tests 10 strains of fungi were obtained forming the fungal consortium, belonging to the following genera: Aspergillus, Aphanoascus, Penicillium, Asteromyces, Gliocladium and Lacellinopsis.

Based on the heavy metal bioleaching tests carried out in the sediments from the Colta's Lagoon, metal concentrations were obtained in the leachate of the fungal treatment, it presents values of 1,522 ppm for Barium, 0,0989 ppm in vanadio and 0,0385 ppm in Copper, based on the data we can say that the native fungi do not have the capacity to biolixivize the heavy metals present in the sediment of Laguna de Colta.
\end{abstract}

Keywords: Bioleaching, heavy metal Barium, heavy metal Vanadium, heavy metal Copper, lake sediment, fungal consortium, Colta`s lagoon.

\section{Resumen}

Se realiza la caracterización físico-química y microbiológica del sedimento, aislamiento y purificación de cepas fúngicas mediante técnicas de siembras sucesivas.

1 Empresa pública metropolitana de gestión integral de residuos sólidos. EMGIRS-EP, Quito-Ecuador, anacris_09@hotmail.es

${ }^{2}$ Universidad Estatal Amazónica, Departamento Ciencias de la Tierra, Puyo-Ecuador, ef.basantesb@uea.edu.ec

3 Escuela Superior Politécnica de Chimborazo, Facultad de Ciencias y Recursos Naturales, Carrera de Ingeniería Ambiental, Carrera de Agronomía, Francisco de Orellana-Ecuador, lcocha@espoch.edu.ec

4 Universidad Técnica de Cotopaxi, Facultad de Ciencias Agropecuarias y Recursos Naturales, Carrera de Ingeniería Ambiental, Latacunga-Ecuador, manuel.clavijo@utc.edu.ec 
Se efectuó la caracterización macroscópica y microscópica de las cepas, se procedió a realizar diferentes pruebas de selección: antagonismo e identificación para su posterior masificación e inoculación en el sedimento, el proceso se ejecutó para la obtención de los consorcios fúngicos nativos de los sedimentos provenientes de la Laguna de Colta. Utilizando equipos como: Cámara de flujo laminar horizontal, autoclave, incubadora, plasma acoplado inductivamente (ICP), estufa, entre los principales.

Al finalizar las pruebas de selección se obtuvo 10 cepas de hongos formando el consorcio fúngico, pertenecientes a los siguientes géneros: Aspergillus, Aphanoascus, Penicillium, Asteromyces, Gliocladium y Lacellinopsis.

En función de las pruebas de biolixiviación de metales pesados realizadas en los sedimentos procedentes de la Laguna de Colta se obtuvieron concentraciones de metales en el lixiviado del tratamiento con hongos presenta valores de 1,522 ppm para Bario, 0,0989 ppm en Vanadio y 0,0385 ppm en Cobre, en base a los datos podemos decir que los hongos nativos no tienen capacidad de biolixiviar los metales pesados presentes en el sedimento de la Laguna de Colta.

Palabras claves: Biolixiviación, metal pesado Bario, metal pesado Vanadio, metal pesado Cobre, sedimento lacustre, consorcio fúngico, laguna de Colta.

\section{Introducción}

Diversas actividades antrópicas e industriales han desencadenado diversos escenarios de contaminación que han deteriorado los ecosistemas, en lo que concierte a medios lacustres son sistemas con mayor vulnerabilidad ya que soportan una alta intensidad de uso del medio, afectando directamente en su proceso de depuración natural. [2]

La Laguna de Colta, uno de los centros turísticos más representativos de la Provincia de Chimborazo por su trascendencia histórica y cultural, se ha convertido en un medio vulnerable a la contaminación, ya que la actividad de dragado de sus sedimentos no contó con orientación y criterios especializados de análisis, provocando un impacto ambiental de considerables repercusiones en ámbitos relacionados con la biodiversidad.

La proliferación de totora, eutrofización y obstrucción de escorrentías han constituido una amenaza en la conservación de este recurso, incidiendo en el proceso de sedimentación y la acumulación de sustancias toxicas en el mismo [1]. En tal virtud, la alteración de su belleza paisajística se ha alterado, hoy peligrosamente el nivel del agua está descendiendo, y es evidente el deterioro del hábitat de los alrededores de la laguna.

La biolixiviación se convierte en una de las técnicas más limpias para reducir la contaminación, mediante el aprovechamiento de microorganismos y su capacidad de transformar o movilizar metales [5]. Por lo que, la presente investigación se fundamenta en la capacidad de la flora microbiana autóctona y su interacción con sustancias contaminantes, a fin de reducir el impacto de los mismos a nivel ambiental y social.

\section{Metodología}

\section{Descripción del área de estudio}

La Laguna de Colta se encuentra ubicada al noroccidente de la Provincia de Chimborazo, en el Cantón Colta, a $17 \mathrm{Km}$ de la ciudad de Riobamba. Tiene un área de 2,5 Km de largo y $1 \mathrm{Km}$ de ancho. Alberga especies nativas de aves y una gran variedad de peces de distintos colores [7]. 


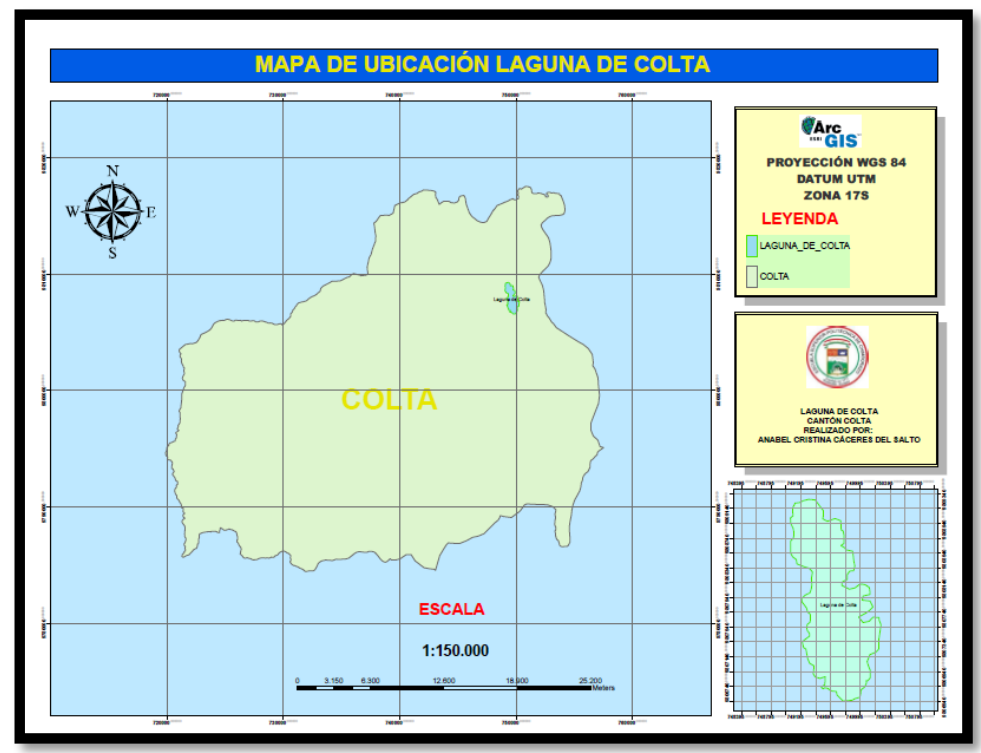

Figura 1. Mapa ubicación Laguna de Colta

Fuente: Elaboración propia

La Laguna cuenta con un área de aproximadamente 204 Ha., dividida en 2 parroquias, Santiago de Quito con un área de 753 Ha., y Sicalpa con 1396 Ha., ambas pertenecen al cantón Colta [7].

\section{Diseño experimental}

Se sujeta a la manipulación de variables, con el objetivo de evaluar la actividad simbiótica de consorcios fúngicos nativos controlando las variables que pueden afectarlos $(\mathrm{pH}$, Humedad, Temperatura, Cantidad de nutrientes).

La investigación se basa en las siguientes características, presenta un nivel descriptivo, la secuencia es de tipo trasversal, el enfoque es cuantitativo, se busca generar conocimiento aplicando un método experimental.

\section{Tratamientos}

El sedimento contaminado proviene de la Laguna de Colta, se tomó un peso total de $15 \mathrm{~kg}$ que fueron distribuidos en recipientes plásticos, en los que se aplicó el TH: Sedimento inoculado con consorcios fúngicos nativos.

\section{Variables de control}

A lo largo del proceso de la investigación, es importante controlar ciertas variables que pueden influenciar en los microorganismos afectando la biolixiviación de los metales:

$-\mathrm{pH}$

- Temperatura

- Humedad

- Crecimiento fúngico [6] [8].

Procesamiento de muestras biológicas

Los hongos presentes en las muestras de sedimentos de la Laguna de Colta requieren nutrientes básicos que son aportados por los medios de cultivo no selectivos como es el caldo nutritivo. 


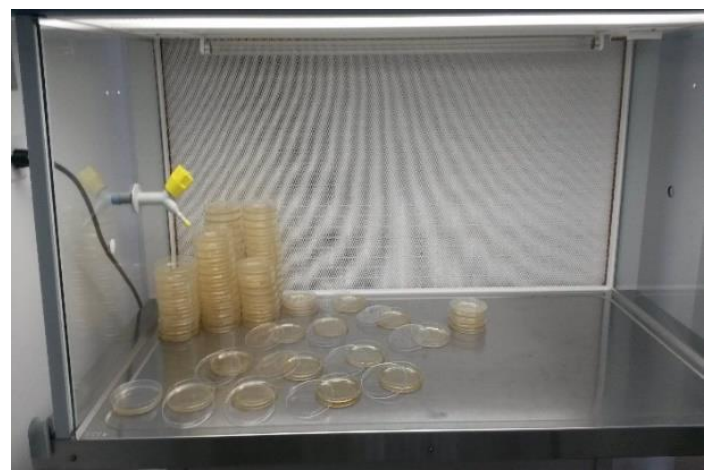

Figura 2. Preparación de cajas petri con agar Fuente: Elaboración propia

\section{Aislamiento y purificación de las cepas fúngicas}

Para la obtención de las cepas fúngicas aptas para el proceso de biolixiviación es necesario purificar mediante un proceso de aislamiento consecutivo [8].

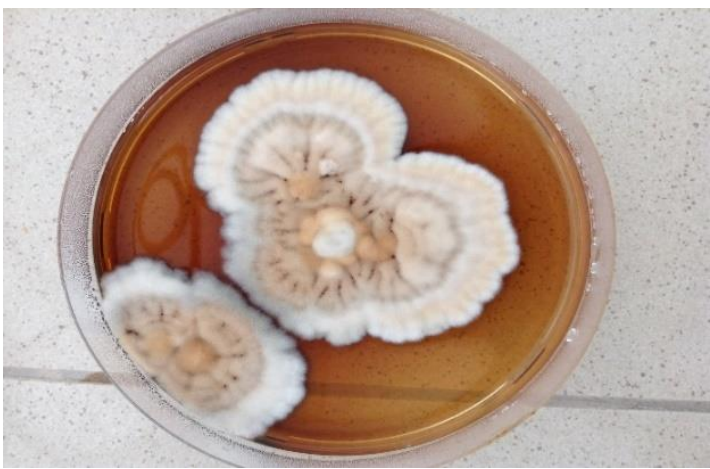

Figura 3. Aislamiento y purificación de cepas fúngicas Fuente: Elaboración propia

\section{Identificación y caracterización de las cepas fúngicas}

La identificación y caracterización permite determinar las características morfológicas de las cepas que vayan a ser seleccionadas para conformar el consorcio en un tiempo determinado. Se realiza una resiembra mensualmente para la conservación de las cepas y evitar el envejecimiento [5].

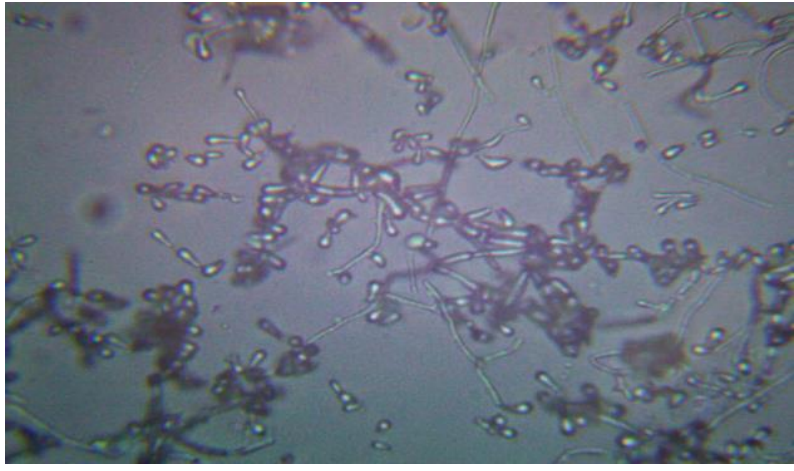

Figura 4. Observación de placas al microscopio Realizado por: Elaboración propia 


\section{Conservación de cepas fúngicas}

La conservación se realizó mediante la elaboración de un banco primario, mismo que permite conservar las cepas por largos periodos de tiempo [4].

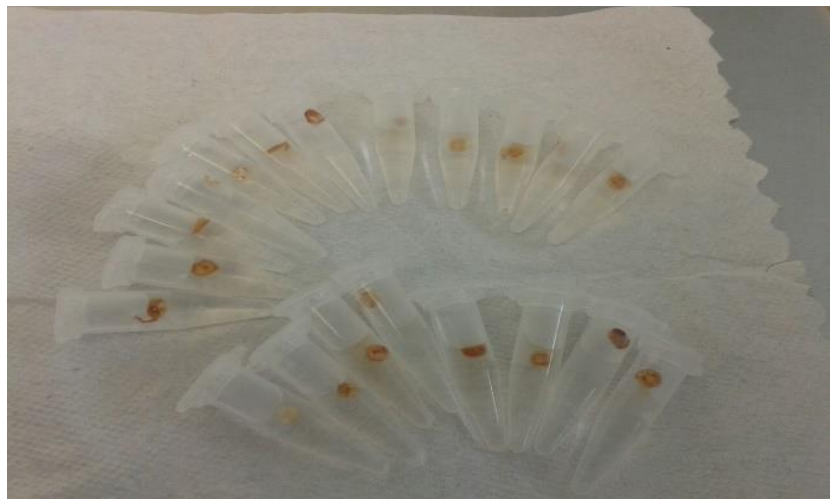

Figura 5. Conservación de cepas fúngicas

Fuente: Elaboración propia

\section{Preparación del inoculo liquido fúngico}

El inoculo liquido de hongos es la sustancia que se colocará en el sedimento para aumentar la carga fúngica del mismo, al ser líquido permitirá que las hifas tengan una mayor superficie de acción con una sola aplicación.

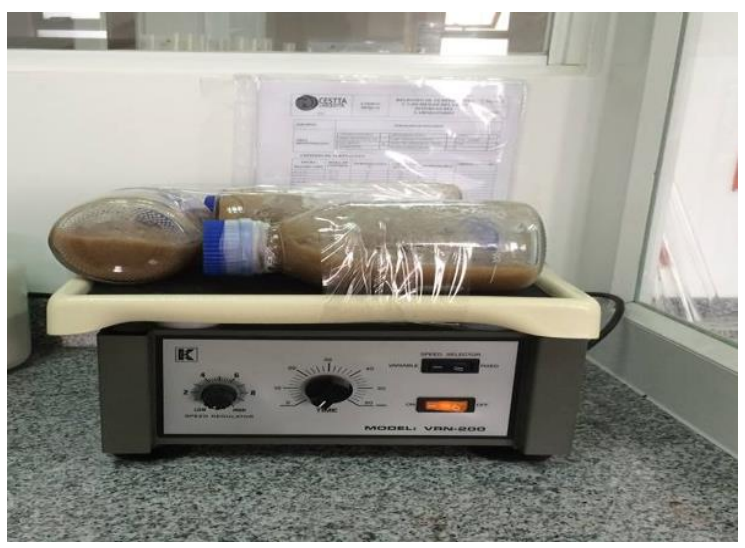

Figura 6. Inóculo fúngico

Fuente: Elaboración propia

\section{Métodos analíticos}

\section{Determinación de metales $(\mathrm{V}, \mathrm{Ba}, \mathrm{Cu})$ por $\mathrm{ICP}$}

Una fuente de ICP está constituido por una corriente de flujo de un gas, específicamente el argón que es ionizado por un campo de radiofrecuencias que oscila a 27,1 MHz, este campo se encuentra enlazado inductivamente al gas ionizado a través de una bobina refrigerada con agua que rodea una "lámpara" de material de cuarzo cuya función es dar soporte y confinar el plasma. Gracias a las elevadas temperaturas del plasma (6 000 a $8000{ }^{\circ} \mathrm{K}$ ) se logra una excitación eficaz de la emisión atómica, permitiendo bajos niveles de detección para muchos elementos, y esto conjuntamente con extenso recorrido dinámico lineal se logra una eficaz detección de multi elementos. Método de referencia: Standars Methods No. 3120 A. [3][9]. 


\section{Determinación de pH}

La medición de $\mathrm{pH}$ de un suelo o de sedimento, se realiza con la ayuda de un potenciómetro y electrodos de vidrio, el principio se fundamente en la medida del potencial eléctrico producido en la membrana de vidrio del mismo, que es producto de las actividades de los iones hidrógeno a ambos lados de la membrana. Método de referencia: EPA 9045 D. 2004

\section{Determinación de la humedad}

Se determina el contenido de humedad gravimétrico del suelo, secando la muestra por medio de la aplicación directa de calor. Método de referencia: Norma ASTM D’4959

\section{Determinación de temperatura}

Los átomos y moléculas por los que está constituida una sustancia no siempre se mueven a velocidad constante, motivo por el cual existe en las moléculas un rango de energía, conocida como energía de movimiento.

La temperatura se define como la medición del calor o energía térmica de las partículas presentes en una sustancia, como se mide su movimiento medio su valor depende del tamaño de las partículas en un objeto y no del número de partículas [10].

\section{Métodos microbiológicos}

La determinación de la carga se la efectúa por medio de la preparación de varias diluciones continuas o sucesivas en un medio de cultivo general nutritivo, para luego sembrarlo en placas petri y conteo [6].

\section{Resultados}

\section{Análisis físico químico del sedimento}

A partir de los resultados obtenidos del análisis físico químico de las muestras recolectadas en la Laguna de Colta, podemos evidenciar que los sedimentos contienen los metales de interés.

Tabla 1. Caracterización Físico Química del Sedimento

\begin{tabular}{|c|c|r|}
\hline PARÁMETROS & UNIDAD & RESULTADO \\
\hline Hidrocarburos Totales & $\mathrm{mg} / \mathrm{Kg}$ & 315,82 \\
\hline Hidrocarburos Aromáticos Policíclicos & $\mathrm{mg} / \mathrm{Kg}$ & 291,63 \\
\hline *Bario & $\mathrm{mg} / \mathrm{Kg}$ & 95,91 \\
\hline *Vanadio & $\mathrm{mg} / \mathrm{Kg}$ & 77,97 \\
\hline *Cobre & $\mathrm{mg} / \mathrm{Kg}$ & 0,63 \\
\hline *Nitrógeno Total & $\%$ & 988,20 \\
\hline *Fósforo Total & $\mathrm{mg} / \mathrm{Kg}$ & 2314,07 \\
\hline *Potasio & $\mathrm{mg} / \mathrm{Kg}$ & 7,08 \\
\hline Potencial de Hidrógeno & Unidades de $\mathrm{pH}$ & 82 \\
\hline *Humedad & $\%$ & \\
\hline
\end{tabular}

Fuente: LAB-CESTTA 


\section{Identificación de cepas fúngicas}

La identificación de las cepas fúngicas se la realizó mediante la técnica de cinta pegante que nos permite observar las características microscópicas de las colonias, esta constituye una de las técnicas más usadas debido a que se conserva la yuxtaposición original de las esporas y segmentos de hifas, y sus estructuras fúngicas no presentan alteraciones significativas. Esta técnica requiere de una persona capacitada en la identificación de las hifas y las esporas de cada muestra que posteriormente son comparadas con una base de datos conociendo así el género de la cepa fúngica.

Tabla 2. Características macroscópicas y microscópicas de las cepas fúngicas.

\begin{tabular}{|c|c|c|c|c|c|c|}
\hline CÒDIGO & GENERO & COLOR DE LA COLONIA & $\begin{array}{l}\text { FORMA } \\
\text { CELULAR }\end{array}$ & ESPORULACIÓN & HIFAS & CRECIMIENTO \\
\hline $\mathrm{LC}-\mathrm{A}$ & Aspergillus & $\begin{array}{l}\text { Centro: Amarillo. } \\
\text { Periferia: Blanco } \\
\text { Verdoso } \\
\end{array}$ & Filamentoso & $\begin{array}{l}\text { Ascosporas } \\
\text { Conidióforo }\end{array}$ & Tabicular & Lento \\
\hline LC - B & Aphanoascus & $\begin{array}{c}\text { Centro y Periferia: } \\
\text { Blanco }\end{array}$ & Filamentoso & Conidióforo & Septadas & Lento \\
\hline $\mathrm{LC}-\mathrm{C}$ & Penicillium & $\begin{array}{l}\text { Centro: Gris verdoso. } \\
\text { Periferia: Blanco }\end{array}$ & Filamentoso & \multirow{3}{*}{ Conidióforo } & \multirow{3}{*}{$\begin{array}{l}\text { Septadas } \\
\text { Hialinas }\end{array}$} & Rápido \\
\hline LC - D & Penicillium & $\begin{array}{l}\text { Centro: Verde-azulado. } \\
\text { Periferia: Blanco }\end{array}$ & Filamentoso & & & Lento \\
\hline LC - E & Penicillium & $\begin{array}{l}\text { Centro: Blanco-Grisáceo. } \\
\text { Periferia: Blanco }\end{array}$ & Filamentoso & & & Rápido \\
\hline $\mathrm{LC}-\mathrm{F}$ & Asteromyces & $\begin{array}{l}\text { Centro: Blanco-Rosáceo. } \\
\text { Periferia: Blanco }\end{array}$ & Filamentoso & Conidióforo & Septadas & Rápido \\
\hline $\mathrm{LC}-\mathrm{G}$ & Penicillium & $\begin{array}{l}\text { Centro: Negro- azulado. } \\
\text { Periferia: Rosado pálido }\end{array}$ & Filamentoso & Conidióforo & $\begin{array}{l}\text { Septadas } \\
\text { Hialinas }\end{array}$ & Lento \\
\hline $\mathrm{LC}-\mathrm{H}$ & Gliocladium & $\begin{array}{l}\text { Centro: Blanco café. } \\
\text { Periferia: Blanco }\end{array}$ & Filamentoso & Conidióforo & $\begin{array}{l}\text { Septadas } \\
\text { Hialinas } \\
\end{array}$ & Lento \\
\hline LC - I & Lacellinopsis & $\begin{array}{c}\text { Centro: Café-verdoso. } \\
\text { Periferia: Blanco- } \\
\text { amarillento } \\
\end{array}$ & Filamentoso & Ascosporas & Septadas & Lento \\
\hline $\mathrm{LC}-\mathrm{J}$ & Aspergillus & $\begin{array}{l}\text { Centro: Negro. } \\
\text { Periferia: Blanco }\end{array}$ & Filamentoso & $\begin{array}{l}\text { Ascosporas } \\
\text { Conidióforo }\end{array}$ & $\begin{array}{l}\text { Septadas } \\
\text { Hialinas }\end{array}$ & Rápido \\
\hline
\end{tabular}

Fuente: Elaboración Propia

En la Tabla 2. Características macroscópicas y microscópicas de las cepas fúngicas se describe el código de cada una de las cepas aisladas, el género y las características morfológicas

Es importante mencionar que en su mayoría las cepas encontradas pertenecen al género Penicillium, de las 10 cepas encontradas en la laguna, fue posible identificar la especie de dos de ellas entre las que tenemos:

- LC - H Gliocladium virens, esta cepa tornó el agar nutritivo de un color café, y puede ser que esta segregue algún tipo de antibiótico. Se conoce que cepas de Gliocladium virens producen un antibiótico de amplio espectro llamado Gliotoxina que mata a muchos microorganismos patógenos del suelo. En la investigación "CONTROL DE CALIDAD EN LA PRODUCCIÓN DEL HONGO Gliocladium 
virens, PATÓGENO DE Anopheles albimanus (Wiedemann 1820), VECTOR DEL PALUDISMO EN MÉXICO "se menciona su potencial como bioinsecticida. (Castillo et al, 2013)

- LC - J Aspergillus niger, considerada la especie más común del género Aspergillus, dentro de los usos que se le da a esta cepa podemos mencionar la obtención de productos químicos como el ácido cítrico, ácido glucónico, así como también de enzimas como la glucoamilasa, galactosidasa.

Prueba de biolixiviación de metales en cada uno de los inóculos conformados Inóculo fúngico.

El consorcio fúngico inoculado en el sedimento está conformado por 10 cepas, correspondientes a 6 géneros Asteromyces, Aspergillus, Aphanoascus, Gliocladium, Lacellinopsis, Penicillium para proceder a la inoculación de esto se preparó un inóculo líquido, a diferencia de las bacterias los hongos no realizan un proceso de oxidación microbiana sino más bien utilizan su metabolismo microbiano como fuente de ácidos que disuelven directamente el metal.

Cada uno de los inóculos se los colocó en 5 lb de sedimento, al mismo que se le añadió Urea para cubrir la demanda de nutrientes de los microorganismos y aserrín que mejora la textura del sedimento, al homogenizar estos compuestos se formó una biopila en un recipiente con una pendiente para recolectar el lixiviado, se coloca el inóculo y posteriormente se realiza la medición del lixiviado en 77 días.

\section{Potencial de biolixiviación}

Para comprobar el potencial de biolixiviación de metales pesados en el sedimento se inocula los consorcios fúngicos en la biopilas y se controla el proceso por un lapso de 77 días, esperando que los metales de interés sean liberados en el lixiviado por efecto de las actividades de los microorganismos. Se verifica la capacidad de los consorcios midiendo la concentración de metales pesados presentes en el lixiviado recogido al final de los 77 días, mismo que fue obtenido a partir del día 14 posterior a la inoculación.

\section{Discusión.}

En la caracterización físico química inicial de los sedimentos se consideró el análisis de los siguientes parámetros, Hidrocarburos Totales de Petróleo (TPH), Metales Pesados de interés Bario (Ba), Vanadio (V), Cobre (Cu), Nutrientes Nitrógeno (N), Fósforo (P) y Potasio (K), además del potencial hidrógeno $(\mathrm{pH})$, y el contenido de Humedad.

Los parámetros que son controlados frecuentemente a lo largo de los 77 días, son $\mathrm{pH}$, humedad, y temperatura, así como también la carga fúngica se controlaron antes y después de cada inoculación.

La concentración de los metales de interés se presenta en la siguiente tabla:

Tabla 3. Concentración de metales pesados en el sedimento

\begin{tabular}{|c|c|c|}
\hline Parámetro & Unidad & Resultado \\
\hline $\mathrm{Ba}$ & $\mathrm{mg} / \mathrm{kg}$ & 291,63 \\
\hline $\mathrm{V}$ & $\mathrm{mg} / \mathrm{kg}$ & 95,91 \\
\hline $\mathrm{Cu}$ & $\mathrm{mg} / \mathrm{kg}$ & 77,97 \\
\hline
\end{tabular}

Fuente: Elaboración propia 
En el país no existe una Norma que controle la concentración de metales pesados en sedimentos lacustres, es por ello, que se determina en función de la concentración del metal en el lixiviado obtenido y por análisis estadísticos.

El volumen de lixiviado recolectado semanalmente, muestra los volúmenes obtenidos por un periodo de 10 semanas. En el tratamiento con hongos en el que se obtuvo un volumen final de $175,06 \mathrm{~mL}$.

Se determinó la concentración de metales presentes en el lixiviado posterior a los 77 días que duró el tratamiento, los valores se muestran en la siguiente tabla:

Tabla 4. Concentración de metales en los lixiviados

\begin{tabular}{|c|c|}
\hline Parámetro & $\begin{array}{c}\text { Concentración A(ppm) } \\
\text { Lixiviado Hongos }\end{array}$ \\
\hline $\mathrm{Ba}$ & 1,522 \\
\hline $\mathrm{V}$ & 0,0989 \\
\hline $\mathrm{Cu}$ & 0,0385 \\
\hline
\end{tabular}

Fuente: Elaboración propia

\section{Evaluación del proceso de biolixiviación}

La eficacia del proceso de biolixiviación se determina mediante una serie de pruebas que permiten comprobar o rechazar la hipótesis planteada, con ayuda del software R-Commander, para el procesamiento de los datos obtenidos.

Influencia del consorcio fúngico

A fin de evaluar la incidencia de los consorcios se aplica el test de Wilcoxon, para analizar la influencia en la concentración antes y después del experimento.

$\boldsymbol{H}_{0}$ : No existe una influencia de los consorcios fúngicos en la concentración de metales antes y después del experimento.

\section{Prueba de Shapiro Wilk}

El valor de p 0.0001094 menor al nivel de significancia (0.05), determina el rechazo de $\mathrm{H}_{0}$ al 95\% de confiabilidad, lo que significa que los datos no provienen de una distribución normal.

\section{Prueba de Wilcoxon}

Aplicamos el test de Wilcoxon para muestras dependientes en una distribución normal, obteniendo un valor de p igual a 0,003906, aceptando $\mathrm{la}_{0}$ al $95 \%$ de confiabilidad, es decir, las concentraciones registradas antes y después de la acción fúngica, factor que incide en la eficacia de los consorcios nativos.

\section{Influencia del tipo de metal sobre la concentración del biolixiviado}

$H_{0}$ : No existe una influencia del metal sobre la concentración del biolixiviado

\section{Prueba de Shapiro Wilk}

Se obtiene un valor de p igual a 0.0001094 que es menor al nivel de significancia (0.05), por lo que, rechazamos $\mathrm{H}_{0}$ al $95 \%$ de confiabilidad, es decir no existe una distribución normal.

\section{Prueba de Kruskall Wallis}

Mediante el análisis para muestras dependientes se obtiene un p valor de 0.05732 aceptando la $\mathrm{H}_{0}$ al $95 \%$ de confiabilidad, determinándose que el tipo de metal no influye en la concentración obtenida en el volumen biolixiviado. 


\section{Influencia del tratamiento sobre la concentración de metales en el lixiviado}

$H_{0}$ : No existe una influencia del tratamiento sobre la concentración de metales en el lixiviado Prueba de Shapiro Wilk

Los datos no provienen de una distribución normal por cuanto el valor de $\mathrm{p}$ es igual a 0.0001094

\section{Prueba de Kruskall Walls}

Se obtiene un p valor de 0,9665 aceptando la $\mathrm{H}_{0}$ al $95 \%$ de confiabilidad, determinándose que el tratamiento mediante consorcios fúngicos nativos no influye en la concentración biolixiviada del metal.

\section{Relación de variables de control}

Las variables de control están relacionadas directamente con la actividad de los microorganismos inoculados en el sedimento, de ahí la importancia de controlar dichos parámetros y de relacionarlos entre sí. Cada parámetro esta interrelacionado, y depende el uno del otro es así que un aumento en la temperatura podría ocasionar perdida de humedad y por tanto afectar la actividad microbiana.

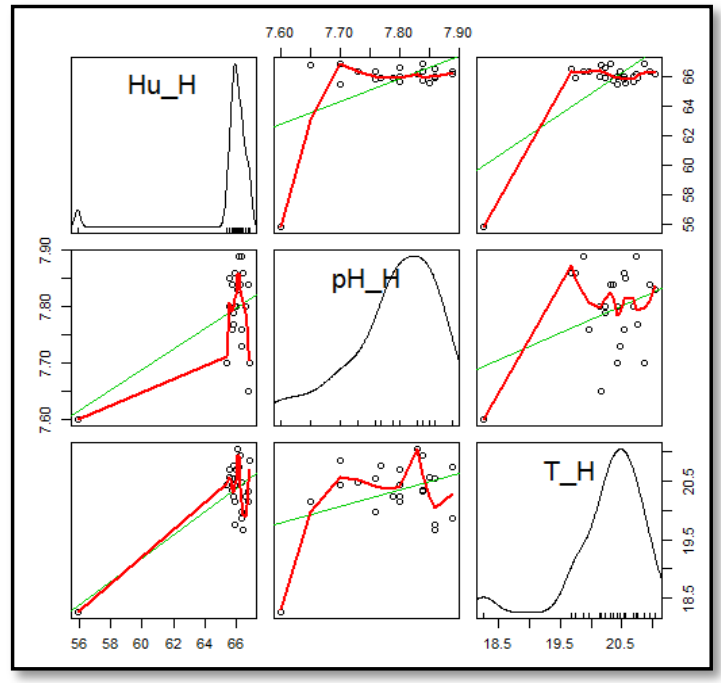

Figura 7. Relación de las variables de control en hongos Fuente: Elaboración propia

En la figura 7: Relación de las variables de control en hongos se observa el comportamiento de los parámetros de control como $\mathrm{pH}$, Humedad y Temperatura durante el proceso de biolixiviación, así como también interrelacionarlos.

\section{Conclusiones}

- La caracterización físico-química inicial del sedimento de la laguna de Colta, demostró la presencia de los metales pesados de interés con las siguientes concentraciones para Bario 291,63 mg/kg, para Vanadio 95,91 mg/kg y para Cobre 77,97 mg/kg.

- Se conformó un consorcio fúngico conformado por 10 cepas entre las que tenemos Aspergillus(LC-A), Aphanoascus(LC-B), Penicillium(LC-C), Penicillium(LC-D), Penicillium(LC-E), Asteromyces(LC-F), Penicillium (LC-G), Gliocladium(LC-H)., Lacellinopsis(LC-I) y Aspergillus(LC-J). 
- El proceso de biolixiviación probado no resultó eficaz para la remoción de metales pesados del sedimento de la Laguna de Colta, ya que los consorcios fúngicos no lograron producir ácidos orgánicos para solubilizar el Bario, Vanadio y Cobre, estos resultados son respaldados por los análisis estadísticos realizados, no tiene la capacidad de biolixiviar los metales de interés, a pesar de mantener en el proceso las condiciones óptimas de crecimiento fúngico.

\section{Referencias bibliográficas.}

[1].ARCHE, Alfredo. Sedimentología del proceso físico a la cuenca sedimentaria 4.ed. Madrid-España CSIC - CSIC Press 2010 pp. 321-322

[2].BARRIOS, Yaima. Biorremediación: una herramienta para el saneamiento de ecosistemas marinos contaminados con petróleo. Revista Biotecnología Aplicada. Vol. 28. Illustrated. La Habana-Cuba. 2011. pp. 61 http://web.b.ebscohost.com/ehost/pdfviewer/pdfviewer?sid=5c746472-7bb9-4953ae4f-b11 cabb3aad6\%40sessionmgr113\&vid=1\&hid=115. 2015-04-14

[3].CASTRO, Gabriel., VALDÉS, Jorge. Concentración de metales pesados $(\mathrm{Cu}, \mathrm{Ni}$, $\mathrm{Zn}, \mathrm{Cd}, \mathrm{Pb}$ ) en la biota y sedimentos de una playa artificial, en la bahía San Jorge $23^{\circ} \mathrm{S}$, norte de Chile. Revista Latin American Journal of Aquatic Research. Vol. 40. Illustrated. Antofagasta-Chile.2012. pp. 268. http://web.a.ebscohost.com/ehost/pdfviewer/pdfviewer?vid=22\&sid=83481c797c8a-44e3-ba3b-5ec2bfe123e9\%40sessionmgr4005\&hid=4214. 2015-03-10

[4].ESPINOSA, Mayra., LUDEÑA, Karina. Lixiviación fúngica de cobre a partir de minerales del distrito minero Portovelo-Zaruma-Minas Nuevas. (TESIS) (Ing. Quím.). Universidad Técnica Particular de Loja. Área Biológica y Biomédica. LojaEcuador. 2007 pp. 27.29

[5].JUAREZ, Argelia. Biolixiviación de minerales sulfuro-ferroso en jales: aislamiento y caracterización de cultivos puros y mixtos de microorganismos involucrados. (TESIS) (Dr. En Ciencias: Área Biotecnología). Universidad de Colima, Área de Ciencias Biológicas y Agropecuarias. Distrito Federal-México.2004. pp. 9-11 http://digeset.ucol.mx/tesis_posgrado/Pdf/Argelia_Juarez_Alcaraz.PDF. 2015-03-06

[6].MIDENCE, Róger., GARCÍA, Josué. Evaluación del potencial de bio-lixiviación fungal autóctona e inoculada de metales en suelos sedimentos contaminados en la región minera de Santo Domingo-Chontales. (TESIS) (Ing. Amb.) Universidad Centroamericana, Facultad de Ciencia, Tecnología y Ambiente, Departamento de desarrollo tecnológico, Managua-Nicaragua.2013 pp. 11-12 http://biorem.univie.ac.at/fileadmin/user_upload/p_biorem/education/research/publi cations/Theses/Tesis_Midence_y_Garcia_2013.pdf. 2015-02-06

[7].SERRANO, Paola. Obtención del consorcio bacteriano nativo del sedimento de la laguna de Colta del cantón Colta. (TESIS) (Ing. Btgia Amb). Escuela Superior Politécnica de Chimborazo, Facultad de Ciencias, Escuela de Ciencias Químicas, Riobamba-Ecuador. 2014. pp. 39-40

[8].TORTORA, Gerad. Introducción a la microbiología. 9.ed. Buenos Aires-Argentina. Medica Panamericana. 2007. pp 78-79, 85-86, 160- 166. 
[9].VOLKE, Tania., et al. Suelos contaminados por metales y metales y metaloides: muestreo y alternativas para su remediación. 2.ed. Distrito Federal-México. Illustrated. 2005. pp. 90-93

[10]. YAGNENTKOVSKY, Nadia. Aplicación de técnicas de biorremediación para el tratamiento de residuos industriales con alto contenido de metales pesados. (TESIS) (Dr. en Ciencias). Universidad Nacional De La Plata, Facultad de Ciencias Exactas, Departamento de Química, Buenos Aires-Argentina. 2011. pp. 5-6 http://sedici.unlp.edu.ar/bitstream/handle/10915/2706/Documento_completo.pdf?se quence $=1$. 2015-02-06

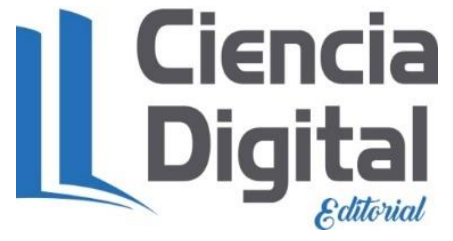




\section{PARA CITAR EL ARTÍCULO INDEXADO.}

Cáceres del Salto, A., Basantes Basantes, E., Cocha Telenchana, L., \& Clavijo Cevallos, M. (2019). Evaluación biolixiviar de metales pesados en los sedimentos de la Laguna de Colta. Ciencia Digital, 3(3.4.), 263-275. https://doi.org/10.33262/cienciadigital.v3i3.4.871

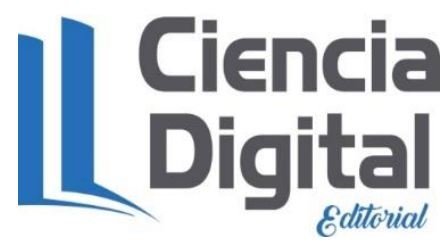

El artículo que se publica es de exclusiva responsabilidad de los autores y no necesariamente reflejan el pensamiento de la Revista Ciencia Digital.

El artículo queda en propiedad de la revista y, por tanto, su publicación parcial y/o total en otro medio tiene que ser autorizado por el director de la Revista Ciencia Digital.
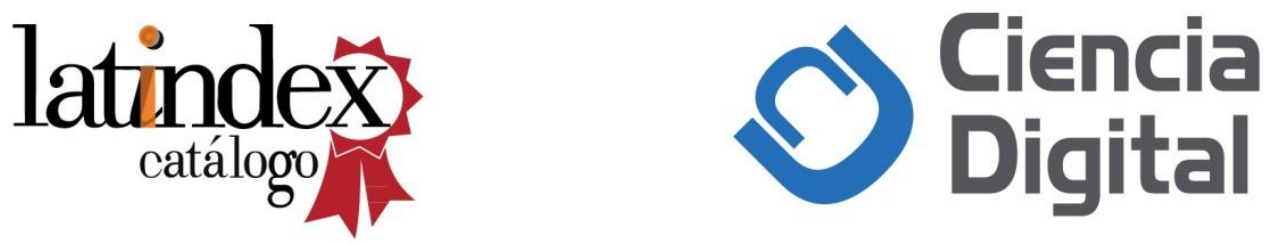